\title{
Editorial
}

\section{Escrever ciência: como o manual da APA pode auxiliar?}

\author{
Rodolfo Augusto Matteo Ambiel \\ Ana Paula Porto Noronba \\ Lucas de Francisco Carvalho \\ Universidade São Francisco - Campinas, SP, Brasil
}

A publicação de uma pesquisa científica é uma das etapas fundamentais do longo processo de se fazer pesquisa. Além de sua importância, há um desafio inerente atrelado à possibilidade de divulgar o estudo científico. De maneira crescente, escrever e publicar ciência vem sendo relacionado com a qualidade da formação dos pesquisadores (veja Linton, Tierney, \& Walsh, 2013), tendência que não está livre de críticas e discussão (e.g., Colpaert, 2012; van Dalen \& Henkens, 2012).

Tantos são os desafios em se escrever e publicar ciência, que levantamentos prévios foram, tratando das principais dificuldades para se aceitar ou recusar artigos científicos (Noble, 2006), sendo tema recorrente em diversas áreas da ciência (e.g., Gastel \& Day, 2016; Masic, 2011). Especificamente em psicologia, o Publication Manual of the American Psychological Association (2010), apesar de ser mais conhecido estritamente como uma diretriz descritiva sobre como "colocar as referências nas normas APA", pode ser considerado uma obra de referência para a estruturação de publicações científicas

O manual da APA, em sua sexta edição, foi desenvolvido e aprimorado ao longo de anos, por profissionais e experts em psicologia, e tem como foco apresentar diretrizes sobre a escrita científica. Essas diretrizes se estendem para definições dos tipos de publicação científica; aspectos éticos e melhores práticas; definições dos elementos que compõem um manuscrito científico; organização das ideias e escolha de palavras, uso do estilo APA (e.g., pontuação, abreviação, números); uso do elementos gráficos de maneira efetiva; regras para citações e normas para referências, e visão geral sobre todas as etapas do processo de publicação.

Como pode ser observado, o tema da publicação científica em psicologia e outras áreas, é complexo e envolve diversos subtópicos para discussão. Um "livro de cabeceira" para qualquer escritor de ciência em psicologia deve ser o manual da APA (2010). A leitura e apropriação de suas diretrizes devem elevar a qualidade dos artigos a serem publicados.

Neste volume 23, número 3, são oferecidos 14 artigos inéditos referentes às diversas temáticas da Psicologia. Agradecemos aos autores e pareceristas pelas contribuições e esperamos que nossos leitores desfrutem desses trabalhos e possam adquirir novos conhecimentos.

O trabalho Theory of Mind Test for Children: Content Validity é o primeiro do presente número. As autoras, Tatina Pontrelli Mecca, Natália Martins Dias, Patrícia Vieira de Oliveira e Monalisa Muniz, tiveram por objetivo investigar as evidências de validade de conteúdo do Teste de Teoria da Mente para Crianças - TMEC.

O foco do trabalho de Maria Isabel da Silva Leme, Laura Carrasqueira Bechara e Mariana Kfouri foi buscar as relações entre estratégias de resolução de conflitos de pré-adolescentes e ocorreência de perdão, além de identificar as suas concepções acerca do ato e sua associação com religião, sexo, entre outros. O título do artigo é $A n$ Exploratory Study of Forgiveness' Place into the Interpersonal Conflicts of Pre-teenagers.

Alessandra Turini Bolsoni-Silva, Rommel Melgaço Barbosa, Alessandra Salina Brandão e Sonia Regina Loureiro verificaram a confiabilidade, sensibilidade e especificidade de um amplo conjunto de indicadores sobre o desempenho acadêmico de universitários. Esse artigo é intitulado Prediction of course completion by students of a university in Brazil.

$\mathrm{O}$ artigo intitulado School Experience During Adolescence: A Comparative Study Between Adolescent Offenders and Not Offenders teve o intuito de testar o modelo da regulação escolar, identificando as variáveis de contextos e mecanismos que se distinguiriam significativamente em adolescents infratores e não infratores. A autoria é de Jorge Luiz da Silva e Marina Rezende Bazon.

Validity Evidence of the Zulliger-SC Test to children's assessment é o estudo desenvolvido por Lucila Moraes Cardoso, Gabriel Vitor Acioly Gomes e Thalita Sena Vieira. Os autores buscaram evidências de validade para o teste de Zulliger pelo Sistema Compreensivo (ZSC).

O objetivo de Valdiney Veloso Gouveia, Hysla Magalhães de Moura, Isabel Cristina Vasconcelos de Oliveira, Maria Gabriela Costa Ribeiro, Alessandro Teixeira Rezende e Tátila Rayane de Sampaio Brito foi verificar a evidências de validade de construto, validade convergente e consistência interna do Questionário de 
Regulação Emociona (QRE). O artigo foi intitulado Emotional Regulation Questionnaire (ERQ): Evidence of Construct Validity and Internal Consistency.

O trabalho intitulado Estratégias de Enfrentamento das Doenças por Idosas Hospitalizadas é de autoria de Laís Claudino Moreira Ribeiro, Verônica Lúcia do Rego Luna e Katruccy Tenório Medeiros. O objetivo das autoras foi analisar as estratégias de enfrentamento de doenças por idosas hospitalisadas.

Andressa Melina Becker da Silva, Wagner de Lara Machado, Anita Colletes Bellodi, Kainara Silva da Cunha e Sônia Regina Fiorim Enumo fizeram contribuições que podem ser consultadas no artigo Jovens insatisfeitos com a imagem corporal: Estresse, autoestima e problemas alimentares. Os autores tiveram por objetivo verificar as relações entre a imagem corporal, estresse percebido, autoestima, Índice de Massa Corporal e comportamento alimentar.

A revisão de literatura Avaliação de candidatos pretendentes no processo de habilitação para adoção: Revisão da Literatura é de autoria de Mariana Silva Cecílio e Fabio Scorsolini-Comin. Os autores objetivaram compreender de que modo a avaliação de pretendentes à adoção no contexto brasileiro vem sendo abordada, descrita e problematizada na literatura científica.

No estudo Evidência de validade da Medida de Atenção Plena pela relação com outras variáveis o objetivo foi buscar evidências de validade da Medida de Atenção Plena (MAP) pela sua relação com outros construtos. O trabalho é de autoria de Jeferson Gervasio Pires, Carlos Henrique Sancineto da Silva Nunes, Maiana Farias Oliveira Nunes, Marcelo Marcos Piva Demarzo, Maria Luiza Bianchi, Mônica Monteiro Kotzias e Giovania Mitie Maesima Cunha.

Daniel Barbieri e Ronaldo Pilati realizaram a pesquisa intitulada Julgamento moral do aborto: influência da atitude prévia, princípios deontológicos e utilitaristas. Os autores objetivaram mensurar o efeito covariante da atutude prévia em relação a um tema moral na relação entre a sobrecarga cognitive e o julgamento de um cenário moral.

Propriedades Psicométricas do Inventário de Fatores Psicológicos de Doenças Relacionadas ao Trabalho: um estudo com trabalhadores de frigorificos teve por intuito avaliar as propriedades psicométricas (validades de critério, construto e fidedignidade) do Inventário de Fatores Psicológicos em Doenças Relacionadas ao Trabalho (IP-T). A autoria é de Romilda Guilland, Roberto Moraes Cruz e Erikson Kaszubowski.

Eunice M. L. Soriano de Alencar, Denise de Souza Fleith, Clarissa Nogueira Borges e Evely Boruchovitch realizaram a pesquisa Criatividade em Sala de Aula: Fatores Inibidores e Facilitadores Segundo Coordenadores Pedagógicos. O objetivo dos autores foi investigar os fatores que dificultam o professor a estimular o desenvolvimento da criatividade discente e procedimentos que o coordenador poderia utilizar para apoiar o docente na promoção da criatividade em sala de aula.

Ao final dessa edição é apresentado o trabalho de Neyfsom Carlos Fernandes Matias, Relações entre Nivel Socioeconômico, Atividades Extracurriculares e Alfabetização. $\mathrm{O}$ autor investigou as implicações do nível socioeconômico (NSE) na alfabetização, na inserção de crianças em atividades oferecidas por organizações não governamentais e os impactos dessas ações no desempenho escolar.

\section{Referências}

American Psychological Association. (2010). Manual of the American Psychological Association - 6th ed. American Psychological Association: Washington, DC.

Colpaert, J. (2012). The "Publish and Perish" syndrome. Computer Assisted Language Learning, 25 (5), 383-391. doi: $10.1080 / 09588221.2012 .735101$

Gastel, B., \& Day, R. A. (2016). How to write and publish a scientific paper - 8th ed. California: Greenwood.

Linton, J. D., Tierney, R., \& Walsh, S. T. (2013). Publish or Perish: How Are Research and Reputation Related? Serials Review, 37 (4), 244-257.

Masic, I. (2011). How to Search, Write, Prepare and Publish the Scientific Papers in the Biomedical Journals. Acta Inform Med, 19 (2), 68-79. doi: 10.5455/aim.2011.19.68-79

Noble, K. A. (2006). Publish or perish: What 23 journal editors have to say. Studies in Higher Education, 14 (1), 97-102. doi: 10.1080/03075078912331377642

Van Dalen, H. P., \& Henkens, K. (2012). Intended and unintended consequences of a publish-or-perish culture: A worldwide survey. Journal of the association for information science and technology, 63 (7), 1282-1293. 